\title{
Preoperative nutritional state is associated with mid- and long-term mortality after cardiac surgery
}

\author{
Krisztina Tóth ${ }^{1 \wedge}$, András Szabó ${ }^{2}$, Ádám Nagy $^{1}$, Dominika Szabó $^{3}$, Balázs Szécsi ${ }^{1}$, Csaba Eke $^{1}$, \\ Ágnes Sándor ${ }^{2}$, Éva Susánszky ${ }^{4}$, Enikő Holndonner-Kirst ${ }^{2}$, Béla Merkely ${ }^{3}$, János Gál ${ }^{2}$, Andrea Székely $^{2}$
}

${ }^{1}$ Doctoral School of Theoretical and Translational Medicine, Semmelweis University, Budapest, Hungary; ${ }^{2}$ Department of Anesthesiology and Intensive Therapy, Semmelweis University, Budapest, Hungary; ${ }^{3}$ Heart and Vascular Center, Semmelweis University, Budapest, Hungary; ${ }^{4}$ Institute of Behavioural Sciences, Faculty of Medicine, Semmelweis University, Budapest, Hungary

Contributions: (I) Conception and design: K Tóth, A Székely; (II) Administrative support: K Tóth, A Szabó, A Székely; (III) Provision of study materials or patients: None; (IV) Collection and assembly of data: K Tóth, A Szabó, D Szabó, A Sándor, É Susánszky, E Holndonner-Kirst, A Székely; (V) Data analysis and interpretation: K Tóth, A Szabó, D Szabó, A Székely; (VI) Manuscript writing: All authors; (VII) Final approval of manuscript: All authors.

Correspondence to: Andrea Székely, MD, PhD, DEAA. Department of Anesthesiology and Intensive Therapy, Semmelweis University, H-1085, Üllöi str. 78, Budapest, Hungary. Email: andi_szekely@yahoo.com.

Background: The frailty score has been developed to determine physiological functioning capacity. The aim of our research was to explore the relationship between frailty factors and mortality in cardiac surgery patients.

Methods: Our research is an observational, single-center, prospective cohort study (registered on ClinicalTrials.gov: NCT02224222), and we studied 69 patients who underwent elective cardiac surgery between 2014 and 2017. Thirty days before the surgery, they completed a questionnaire that contained questions related to social support, self-reported life quality-happiness, cognitive functions, anxiety and depression. Demographic, anthropometric and medical data were widely collected. The Geriatric Nutritional Risk Index (GNRI) and the Comprehensive Geriatric Assessment (CGA)-based frailty index were calculated as a sum and the domains, respectively. Cox regression and the Kaplan-Meier tests were applied to analyze survival and relative risk. The primary outcome was mid-term mortality.

Results: The patients' mean age was 65.43 years [standard deviation (SD): 9.81 years]. The median followup was 1,656 days of survival [interquartile range (IQR), 1,336-2,081 years], during this period 14 patients died. The median of EuroSCORE II was 1.56 (1.00-2.58) points. The median preoperative albumin level was $32.80 \mathrm{~g} / \mathrm{L}$ (IQR, 29.9-35.8 g/L). Major adverse cardiovascular and cerebral events (MACCEs) occurred 7 times during follow-up. The nutrition score of the CGA was significantly associated with worse long-term survival [score; hazard ratio (HR): 5.35; 95\% CI: 1.10-25.91, P=0.037]. After adjustment for EuroSCORE II and postoperative complications the noncardiovascular CGA score was associated with overall mortality [adjusted hazard ratio (AHR): 1.44, 95\% CI: 1.02-2.04, P=0.036]. In the multivariable Cox regression, GNRI <91 showed an increased risk for mortality (AHR: 4.76, 95\% CI: 1.52-14.92, P=0.007).

Conclusions: The CGA-based noncardiovascular score and nutritional status should be assessed before cardiac surgery prehabilitation and may help decrease long-term mortality.

Keywords: Frailty; frailty score; nutrition; Geriatric Nutritional Risk Index (GNRI); cardiac surgery

Submitted Apr 25, 2021. Accepted for publication Jul 15, 2021.

doi: 10.21037/apm-21-1015

View this article at: https://dx.doi.org/10.21037/apm-21-1015

\footnotetext{
^ ORCID: 0000-0001-8660-8583.
} 


\section{Introduction}

Several well-developed stratification systems exist to estimate outcomes after surgery $(1,2)$. Clinical experience suggests that there should be other factors influencing postoperative outcome in addition to routine physiological risk stratifications. These are likely to be social, functional and psychological $(3,4)$. Over the past two decades, researchers have begun to address frailty, which is used to describe a multidimensional syndrome of reserve deficiency (cognitive function, health status, physical ability), although it has not been easy to standardize. Our previous report studied psychological factors, such as dementia, depression, and anxiety, in cardiac surgery patients, in which low levels of education and anxiety were associated with mid-term mortality (5). Recently, examination of patients' physical status, such as nutritional status, has been needed. Program designers can use the information about frailty to determine the range of services that might be required and satisfies the estimated need for them. Stratification of frailty can help in planning interventions or the prediction of a patient's risk of adverse outcomes or the need for hospital or rehabilitation care (6). For morbidity, mortality, postoperative complications and length of hospital stay frailty are an independent risk factors in the field of surgery (7).

The short- and mid-term survival of frail patients who have undergone cardiac surgery is significantly worse than the non-frail population (8-11).

Currently, there are a variety of scoring systems that calculate frailty, one of which is the comprehensive geriatric assessment (CGA). It is a multidimensional, multidisciplinary diagnostic and therapeutic process to determine the medical comorbidities, functional status, cognitive function, emotional condition, nutritional status, frequent use of multiple drugs, and geriatric syndromes (urinary incontinence, risk of falling, delirium, visual or hearing difficulties) and to develop a harmonized treatment and follow-up plan $(12,13)$.

In older people's nutritional status, many factors play an important role, such as increased drug use, dementia, loss of appetite, swallowing difficulties, and social isolation (14). Geriatric patients are more sensitive to malnutrition, which causes an increased risk of mortality and infection, a prolonged hospital stay, and admission to aged care facilities $(15,16)$. The Geriatric Nutritional Risk Score (GNRI) is a numerical value based on body weight and serum albumin (17).

\section{The aim of this study}

We aimed to discover the usefulness of CGA and GNRI in addition to the assessment of depression and anxiety in patients undergoing elective cardiac surgery. Furthermore, the study population was compared to the Hungarian population.

We present the following article in accordance with the STROBE reporting checklist (available at https://dx.doi. org/10.21037/apm-21-1015).

\section{Methods}

This prospective cohort study was registered on ClinicalTrials.gov (25/08/2014; NCT02224222) and approved by the Regional Ethics Committee (TuKEB 250/2013). Patients were invited to be part of this study, while they were at the anesthesia consultation. All methods were conducted in accordance with the relevant guidelines and regulations (Declaration of Helsinki). Five to 30 days before the elective surgery the baseline questionnaires were collected. The inclusion criteria were: age over 18 years, native Hungarian speaker and elective cardiac surgery. Exclusion criteria were: pregnancy, legal incapacity or limited ability to understand the procedures and ethical approval. All patients were able to make a choice to participate in the study and gave written informed consent accordingly. Eighty-five adult patients provided informed consent and were enrolled prospectively at the Department of Cardiac Surgery of the Heart and Vascular Centre of Semmelweis University, Budapest between September 2014 and August 2017. Because of surgical cancellation, 6 patients were excluded. Eight participants were unable to complete the tests because they had a different surgery type, and two additional participants were excluded due to heart transplantation or transcatheter aortic valve implantation (Figure 1).

\section{Data collection and scoring systems}

Clinical factors, such as preoperative medical history, perioperative laboratory parameters (blood counts, ion homeostasis, liver function, renal function, etc.) (18), intraoperative variables (operation time, cross-clamp time, blood loss, fluid balance), postoperative factors [fluid balance, intensive care unit (ICU) stay, hospital stay, medications, etc.] were collected, outcomes, incidence 


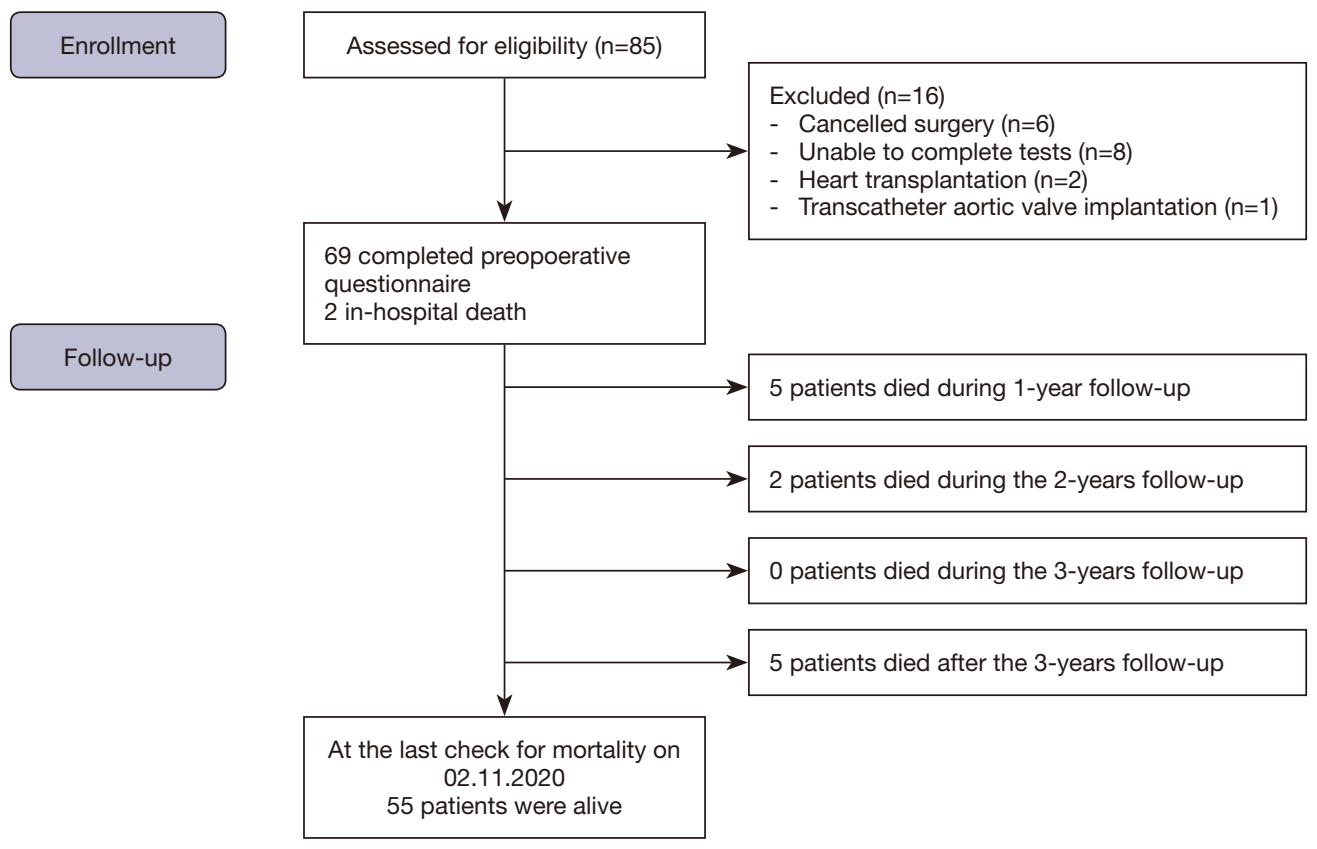

Figure 1 Follow-up. The picture above shows that between September 2014 and August 2017, 85 adult patients provided informed consent and were enrolled prospectively at the Department of Cardiac Surgery of the Heart and Vascular Centre of Semmelweis University, Budapest, Hungary. Six patients were excluded because of surgical cancellation. Eight participants were unable to complete the tests because they had a different surgery type, and two additional participants were excluded due to heart transplantation or transcatheter aortic valve implantation.

and quality of postoperative complications [major cerebrovascular or neurological event (MACCE) (19); multi-organ failure, cardiac arrest, sepsis etc. based on the STS definition references (20)] were compiled. The international normalized ratio (INR) excluded Model for End-Stage Liver Disease (MELD) score (MELD-XI) was calculated as follows:

$5.1 * \ln \left(\right.$ totalbilirubin $\left.\left(\frac{m g}{d l}\right)\right)+11.76 * \ln \left(\right.$ creatinine $\left.\left(\frac{m g}{d l}\right)\right)+9.44$

Values below 1 are taken as 1 to avoid a negative result. Many cardiovascular patients undergo oral anticoagulation therapy, which greatly affects the INR and distorts the result. In cases where the patient's albumin level was not available, $65 \%$ of the total protein level was used. The medical record was used to capture comorbid conditions and illness severity and underlies the calculation of cardiac surgery risk scores. These risk scores are widely used to predict the outcomes of patients undergoing cardiac surgery. The risk scores used in this study were the logistic EuroSCORE (21) and the American Society of
Anaesthesiologist risk score (ASA score) $(22,23)$.

\section{Psychosocial factors}

Patients were asked to complete the following questionnaires regarding psychosocial factors: the Spielberger State-Trait Anxiety Inventory (STAI-S, STAI-T), the Beck Depression Inventory (BDI), the Devins Illness Intrusiveness Rating Scale, the Geriatric Depression Scale, the Mini Mental State Examination (MMSE), the Somatic Symptom Severity Scale, the Caldwell Social Support Dimension Scale (CSSDS), and specific parts of the Hungarostudy (HS) survey [a representative national study from 2013, used as a control group, measuring biopsychosocial background, diseases and health-related quality of life (HRQ)]. The details of these questionnaires have been previously described (24).

Self-reported satisfaction and happiness questionnaire was completed by the participants using a one to ten scale. These values were noted earlier as crucial aspects of defining the long-term mortality of healthy adult volunteers $(25,26)$.

Anxiety was determined by using two scales from 
the Spielberger State-Trait Anxiety Inventory (STAI-S, STAI-T), which examines the factors that influence information and stimulus processing. Current anxiety and the tendency toward anxiety were examined (27). In the Hungarian population, the STAI has well-documented high validity and reliability (28).

The BDI measures affective disorders. The BDI is a self-administered 21-item cognitive questionnaire and is a conventional tool for mapping depression (29). In the Hungarian population, the validity and reliability of the BDI are high (30).

To analyse the effect of the patient's illness on different social issues, the Devin's Illness Intrusiveness Rating Scale was used. This 13-item questionnaire examines lifestyle disorders caused by illness, activities and interests that can jeopardize good psychosocial health status and contribute to emotional distress in chronic diseases (31).

The Geriatric Depression Scale (GDS) is a short, selfadministered questionnaire with simple yes-or-no questions that contains 30 items to assess the appearance of depression in the elder population. A short form of the GDS, which includes fifteen questions, was used (32).

Cognitive function was screened with the Mini-Mental State Examination (MMSE) questionnaire. The MMSE is a cognitive test that is suitable to screen for signs of dementia. The test assesses a wide range of areas, covering short-term memory, auto- and allopsychic orientation, language use, arithmetic computation and basic visual-motor skills (33).

The Somatic Symptom Severity Scale [Patient Health Questionnaire 15 (PHQ15)] examines various symptoms, such as dizziness, gastrointestinal disorders, dyspnoea and chest pain. In addition, 2 parts from the mood section (exhaustion and sleep) are scored 0 ("not at all"), 1 ("several days") or 2 ("more than half the days" or "nearly every day") (34).

The brief form of the Athens Insomnia Scale Inventory (AIS-5) was used, and moderate or severe insomnia was also documented. AIS- 5 is $\geq 4$ was the cut-off score, which is indicating possible insomnia (35).

Finally, the CSSDS measures the patient's social web structure. The bond of different interpersonal relationships and how much the participant can count on those around them were studied. A familial (grandparents, parents, spouse, children and other relatives) and nonfamilial (neighbor, schoolmate, colleagues, other clerical or social company) support score was established (36,37).

Our dataset was compared to the HS population. Nationally representative, free-access, face-to-face household surveys are carried out in Hungary every 10 years, most recently in $2013(\mathrm{n}=2,000)(38,39)$. The HS consists of basic questions about age, gender, education, physical status, marital status, religion, and the inventories listed above. Furthermore, it includes the BDI, AIS, STAI, CSSDS, PHQ15 and Devin's Illness Intrusiveness Rating Scale. In the HS, additional questions were asked about alcoholic beverage consumption, smoking habits and income. A shorter form of the HS 2013 was used in our study to compare the two populations. The propensity score matching method was used to compare the identical questions. The data was compared based on age, sex, residence and employment state.

\section{CGA-based frailty}

Historically, data collection for the frailty scoring system has not been routinely performed by the patient's treatment team. The frailty score was calculated for each participant at the time of preoperative assessment. The score was calculated only after the patient selection has been completed. The medical charts were used to obtain an accurate patient history on both cardiac and noncardiac comorbidities. Furthermore, these were used to collect the pre- and post-operative laboratory results. During the preoperative questionnaire patients' living situation and their social assistance were asked (17). The frailty score (range, 0-41) was based on the CGA model of frailty. It was calculated by the proportion of deficits among 41 items spanning the following 4 domains: medical history, functional limitations, cognition, and nutrition.

Medical history (cardiovascular, noncardiovascular and medication), functional limitations (Nagi items, RosowBreslau items), cognitive function, and nutritional status were used. The cardiovascular score includes angina, hypertension, atrial fibrillation or flutter, congestive heart failure, diabetes, myocardial infarction, peripheral vascular disease, coronary artery disease, and stroke or transient ischaemic attack (TIA). The noncardiovascular score contains anxiety, asthma, cancer diagnosed within 5 years or metastatic cancer, arthritis, chronic kidney disease [glomerular filtration rate (GFR) $<60 \mathrm{~mL} / \mathrm{min}$ ], chronic obstructive pulmonary disease (COPD), degenerative spine disease, depression (GDS >9), falls in the past year, and sensory impairment. The premedication score reports the use of $\geq 5$ prescription drugs before the surgery. Nagi items include difficulty stooping, crouching or kneeling and difficulty lifting or carrying $4.5 \mathrm{~kg}$. Rosow-Breslau items are the incapability of walking up or down a flight of stairs 
and the incapability of doing heavy work around the house. Cognitive function was calculated based on the MMSE score in the following four groups: <21, 21-23, 24-26, and 27-30 points. Nutritional status includes serum albumin $<35.0 \mathrm{~g} / \mathrm{L}$, body mass index $(\mathrm{BMI})<21 \mathrm{~kg} / \mathrm{m}^{2}$, unintended weight loss $>4.5 \mathrm{~kg}$ in the past year (13).

GNRI was calculated based on the following equation:

$$
\begin{aligned}
\text { GNRI }= & 14.89 \times \text { serumalbumin }\left(\frac{g}{d L}\right) \\
& +41.7 \times\left(\frac{\text { preoperativebodyweight }(\mathrm{kg})}{\text { standardbodyweight }(22 \times \text { height } \times \text { height }(\mathrm{m}))}\right)
\end{aligned}
$$

and the cut-off was $91(40,41)$ which means at risk patients were $<91$.

\section{Outcomes}

The primary outcome was mortality, last assessed on November 2, 2020. Annual mortality up to 3 years was also examined. We compared the study population to the Hungarian population with data retrieved from the HS database.

\section{Statistical analysis}

All enrolled patients were included in the analysis set. SPSS version 22.0. (IBM Corp. Released 2013. IBM SPSS Statistics for Windows, NY, USA) statistical software was used to perform statistical analyses, and PSMATCHING3 extension that is relying on published packages in $\mathrm{R}$.

Continuous variables were described as median and interquartile range (IQR, 25 th to 75 th percentiles) or as mean and standard deviation (SD). Categorical variables were presented as number (n) and percentage. To determine the type of distribution the Kolmogorov-Smirnov test and the Shapiro-Wilk test were used. For categorical variables, the Chi-square-test was used; for continuous variables, nonparametric analysis was used, with the MannWhitney $U$ test as the default. Categorical variables were calculated from continuous scales with cut-off values based on international literature. Univariate and multivariable Cox regression analyses were also performed. To find differences in short-, mid- and long-term survival analyses Breslow tests and Kaplan-Meier analysis with log-rank were used. To investigate the correlation, Pearson's correlation was used. Statistically significant was considered $\mathrm{P}<0.05$. Between the Hungarian patient cohort and our cardiac population, a matched analysis was performed by propensity for comparative analysis. In propensity score matching, pairs were created from the HS representative group and the cardiac surgery group according to age, sex, place of residence and legal status. Between the treated and control groups the balance on baseline covariates was assessed using absolute standardized differences. A value less than 0.25 was considered an acceptable standardized bias. As the pairs were created, identical questions were compared to analyze the difference in psychological attitude and social state between the general and surgical populations.

\section{Results}

\section{Descriptive and outcome data}

Sixty-nine patients' data were analyzed. The mean age was $65.33(\mathrm{SD} \pm 9.83)$ years. Forty-four $(63.8 \%)$ patients were male. During the follow-up (1,656 days, IQR, 1,3362,081 days), 14 patients (20.3\%) died. In the postoperative period, the median length of ICU stay was 2.0 days (IQR, 1.0 3.0 days). The median length of hospital stay was 10.0 days (IQR, 8.0-14.0 days). There were two in-hospital deaths, $7.2 \%$ (5 patients) in the first year, and $2.9 \%$ ( 2 patients) in the second year.

The median ASA score was 3.00 (2.00-3.00), while the median EuroSCORE II was 1.56 (1.00-2.58). The median operation time was $180.0 \mathrm{~min}(153.50-213.75 \mathrm{~min})$. Twenty-five patients underwent coronary artery bypass grafting (CABG), 30 underwent valve operations, and 14 underwent combined operations.

The demographic and perioperative variables are shown in Table 1. Those who died were significantly older, and had lower serum albumin, GFR and sodium levels than those in the survivor group. There was no significant difference in C-reactive protein (CRP) levels and white blood cell (WBC) levels between the deceased and survivor groups. Between the two groups mentioned earlier there were no significant difference in the BMI or MELD-XI scores.

We examined the nutritional status of the target population. Univariate Cox regression on the CGA-based nutritional score showed a correlation between 1 year (HR: 6.74, 95\% CI: 1.30-34.81, $\mathrm{P}=0.023$ ) and 2 years (HR: 5.37, 95\% CI: $1.10-26.08, \mathrm{P}=0.037)$.

We demonstrated by univariate Cox regression that the risk of short- and long-term mortality was higher in those who had fewer than 91 GNRI points (HR: 5.75, 95\% CI: 1.98-16.66, $\mathrm{P}=0.001)$. Multivariate Cox regression showed 
Table 1 Preoperative variables and overall mortality

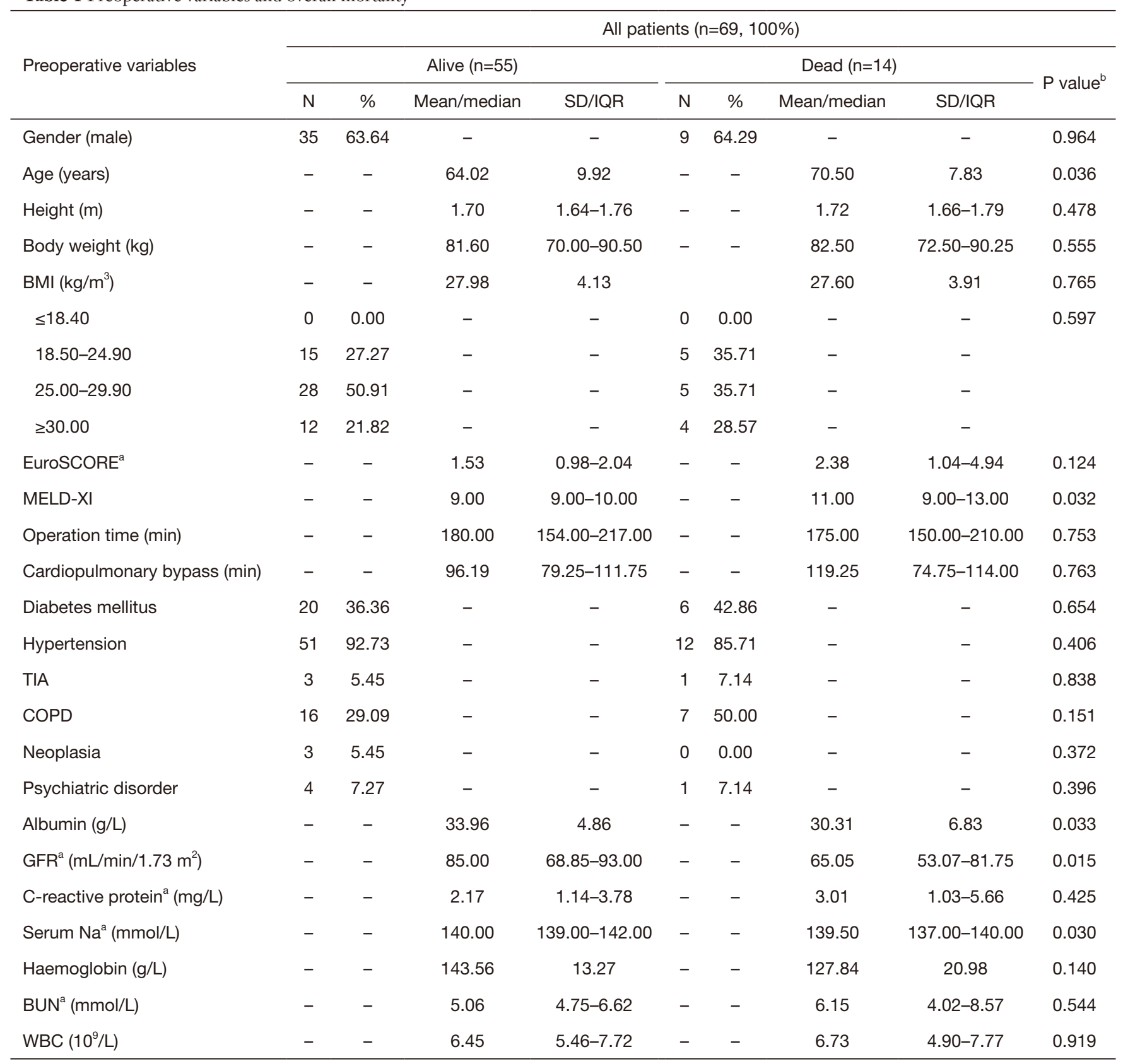

${ }^{a}$, not normal distribution; ${ }^{b}$, Pearson chi square test for categorical variables and Man-Whitney $\mathrm{U}$ test for continuous variables. BMI, body mass index; BUN, blood urea nitrogen; COPD, chronic obstructive pulmonary disease; EuroSCORE, European System for Cardiac Operative Risk Evaluation; GFR, glomerular filtration rate; MELD-XI, Model for End-Stage Liver Disease excluding international normalized ratio; TIA, transient ischaemic attack; WBC, white blood cell.

that GNRI below 91 was associated with mid- (HR: 4.35, 95\% CI: 1.05-17.98, $\mathrm{P}=0.042$ ) and long-term (HR: 4.76, 95\% CI: $1.52-14.92, \mathrm{P}=0.007)$ survival after adjustment for postoperative complications and EuroSCORE II. A KaplanMeier curve is shown in Figure 2.
We adjusted both the nutritional score based on CGA (HR: 5.65, 95\% CI: 1.16-27.52, P=0.032) and GNRI (HR: $0.16,95 \%$ CI: $0.05-0.47, \mathrm{P}=0.001$ ) for MELD-XI, and it seems that these scores are independent risk factors for short- and long-term mortality. We also examined the 


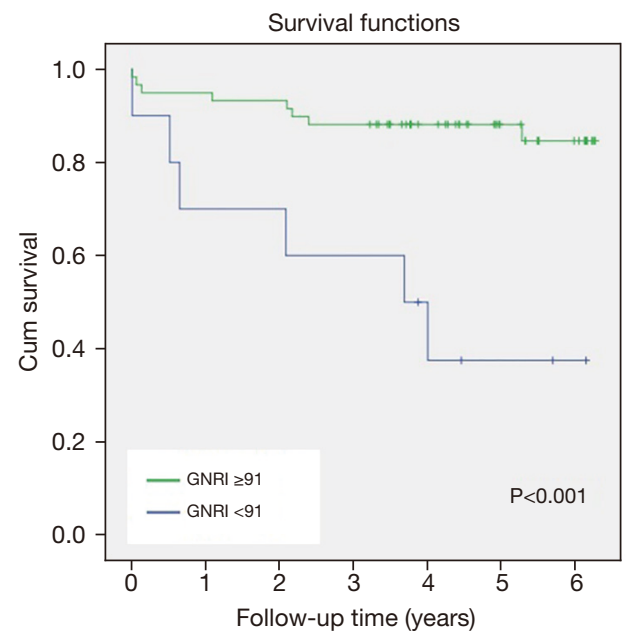

Figure 2 Kaplan-Meier survival based on the GNRI score (green: GNRI $\geq 91$; blue: GNRI <91). The picture above shows a log-rank test in a Kaplan-Meier curve. Follow-up time is given in years. GNRI, Geriatric Nutritional Risk Index.

short-term relationship between the nutritional score based on CGA (AHR: 7.02, 95\% CI: 1.30-37.83, $\mathrm{P}=0.023$ ) and GNRI (AHR: 9.54, 95\% CI: 1.27-71.51, $\mathrm{P}=0.028$ ) and BMI, which also suggests independence. We adjusted the GNRI to the BMI score in the long term (AHR: 12.19, 95\% CI: 2.81-52.85, $\mathrm{P}=0.001$ ), which was a significant risk factor (Table 2). BMI was not associated with mortality, and there were no correlations between albumin and BMI or between nutritional score and BMI. BMI and GNRI have a negative moderate correlation (Table 3).

A higher noncardiovascular score based on CGA showed a $42 \%$ increased risk for mortality (AHR: $1.42,95 \%$ CI: 1.03-1.96, $\mathrm{P}=0.029)$. In the multivariate Cox regression, the noncardiovascular score also showed a significantly higher risk of total mortality after adjustment for postoperative complications and the EuroSCORE (AHR: 1.44, 95\% CI: 1.02-2.04, $\mathrm{P}=0.036)$. CGA functional items such as Nagi and Rosow-Breslau, MMSE, and cardiac score assessed by the CGA were not related to mortality (Table 4).

\section{Hungarostudy}

The cardiac surgery group was compared with the HS group. Sixty-three pairs were made using the propensity score matching analysis. The study population reported smoking habits or financial difficulties less frequently than the HS cohort. The comparison based on the CSSDS suggests that the study population receives less social support outside of their families than the HS group. The balance calculations after propensity score matching analysis are shown in Table 5, and the socioeconomic comparison of the matched cohorts are shown in Tables 5,6.

\section{Discussion}

We found that noncardiovascular medical history and nutritional status were associated with higher mortality after adjustment for EuroSCORE II and postoperative complications. Cognitive state, functional status and cardiac risk factors assessed by the CGA frailty tool were not related to mortality. An independent relationship existed if the model was adjusted for the MELD score, a scoring system for estimating hepatic and renal dysfunction. Nutritional and noncardiovascular domains of CGA can be used in cardiac surgical populations for mid-term mortality prediction. The nutritional score is an independent predictor after adjustment for BMI in short- and mid-term survival.

After cardiac surgery frailty has been acknowledged as a risk factor for a poor prognosis and the importance of the scoring system is emphasized in different cohorts of patients who underwent major surgery (10). The most appropriate method for the evaluation of frailty is still lacking. Two approaches that have been used frequently are the deficit accumulation and phenotypic approaches.

Fried and colleagues developed a phenotypic model that concentrate on patient characteristics across the following 5 items: unintended weight loss, self-reported fatigue, slow gait speed, reduced grip strength and low physical activity (42). The deficit aspect was first used by the Canadian Study of Health and Aging, and the survey contains 70 different physical, mental, medical and functional problems (6). Considering 30-day morbidity, mortality and failure to rescue, the risk was significantly increased for those who had dependence in daily living (43).

The CGA uses a multidisciplinary diagnostic method to examine medical, psychological, functional, and social capabilities. Moreover, it can be used if certain domains in the assessment were not done (13). CGA-based frailty index (FI-CGA) was compiled based on the previously standardized CGA and population clinical trials, also in cardiology (44). The FI-CGA was associated with a higher risk of death and hospital readmissions (45-47). In our study population, the parts of the frailty assessment that deal with difficulties in everyday movements like kneeling, writing 
Table 2 Noncardiovascular score, nutritional score and GNRI's univariant and multivariant Cox regression

\begin{tabular}{|c|c|c|c|c|c|c|c|c|c|}
\hline \multirow{2}{*}{ Follow-up } & \multirow{2}{*}{ Scores } & \multicolumn{4}{|c|}{ Univariant Cox regression } & \multicolumn{4}{|c|}{ Multivariant Cox regression } \\
\hline & & $\operatorname{Exp}(B)$ & Lower & Upper & $P$ value & $\operatorname{Exp}(B)$ & Lower & Upper & $P$ value \\
\hline \multirow[t]{5}{*}{ 1-year mortality } & Non-cardiovascular Score & 1.16 & 0.71 & 1.90 & 0.530 & 1.21 & 0.71 & 2.07 & 0.488 \\
\hline & Nutritional Score & 6.74 & 1.30 & 34.81 & 0.023 & 15.05 & 2.26 & 100.17 & 0.005 \\
\hline & GNRI & 4.86 & 1.09 & 21.78 & 0.039 & 3.44 & 0.65 & 18.17 & 0.150 \\
\hline & $\mathrm{GNRI}^{\mathrm{a}}$ & & & & & 5.64 & 1.21 & 26.39 & 0.028 \\
\hline & $\mathrm{GNRI}^{\mathrm{b}}$ & & & & & 9.54 & 1.27 & 71.51 & 0.028 \\
\hline \multirow[t]{4}{*}{ 2-year mortality } & Nutritional Score & 5.37 & 1.10 & 26.08 & 0.037 & 3.64 & 0.51 & 25.78 & 0.197 \\
\hline & GNRI & 5.51 & 1.47 & 20.57 & 0.011 & 4.35 & 1.05 & 17.98 & 0.042 \\
\hline & $\mathrm{GNRI}^{\mathrm{a}}$ & & & & & 5.93 & 1.56 & 22.58 & 0.009 \\
\hline & $\mathrm{GNRI}^{\mathrm{b}}$ & & & & & 10.38 & 1.74 & 61.82 & 0.010 \\
\hline \multirow[t]{7}{*}{ Total mortality } & Cardio Score & 0.94 & 0.61 & 1.44 & 0.778 & 0.78 & 0.47 & 1.28 & 0.330 \\
\hline & Noncardiovascular Score & 1.42 & 1.03 & 1.96 & 0.029 & 1.44 & 1.02 & 2.04 & 0.036 \\
\hline & Premedication Score & 1.20 & 0.41 & 3.46 & 0.733 & 1.07 & 0.36 & 3.21 & 0.904 \\
\hline & Nagi Score & 1.02 & 0.44 & 2.34 & 0.963 & 1.01 & 0.47 & 2.60 & 0.820 \\
\hline & Rosow Score & 1.67 & 0.68 & 4.06 & 0.259 & 1.69 & 0.65 & 4.35 & 0.280 \\
\hline & $\mathrm{GNRI}^{\mathrm{a}}$ & & & & & 6.17 & 2.10 & 18.13 & 0.001 \\
\hline & $\mathrm{GNRI}^{\mathrm{b}}$ & & & & & 12.19 & 2.81 & 52.85 & 0.001 \\
\hline
\end{tabular}

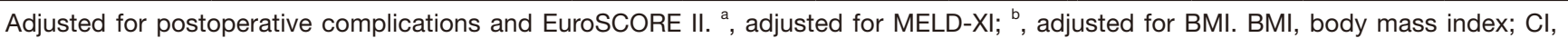
confidence interval; GNRI, Geriatric Nutritional Risk Index; MELD-XI, Model for End-Stage Liver Disease excluding international normalized ratio.

Table 3 BMI correlation to nutritional parameters

\begin{tabular}{lcc}
\hline Nutritional parameters & \multicolumn{2}{c}{ BMl correlation } \\
\cline { 2 - 3 } & Pearson correlation & P value \\
\hline Albumin $(\mathrm{g} / \mathrm{L})$ & 0.09 & 0.434 \\
Nutritional score & -0.12 & 0.343 \\
GNRI & -0.41 & $<0.001$ \\
\hline
\end{tabular}

BMI, body mass index; GNRI, Geriatric Nutritional Risk Index. 
Table 4 Psycho-social questionnaires

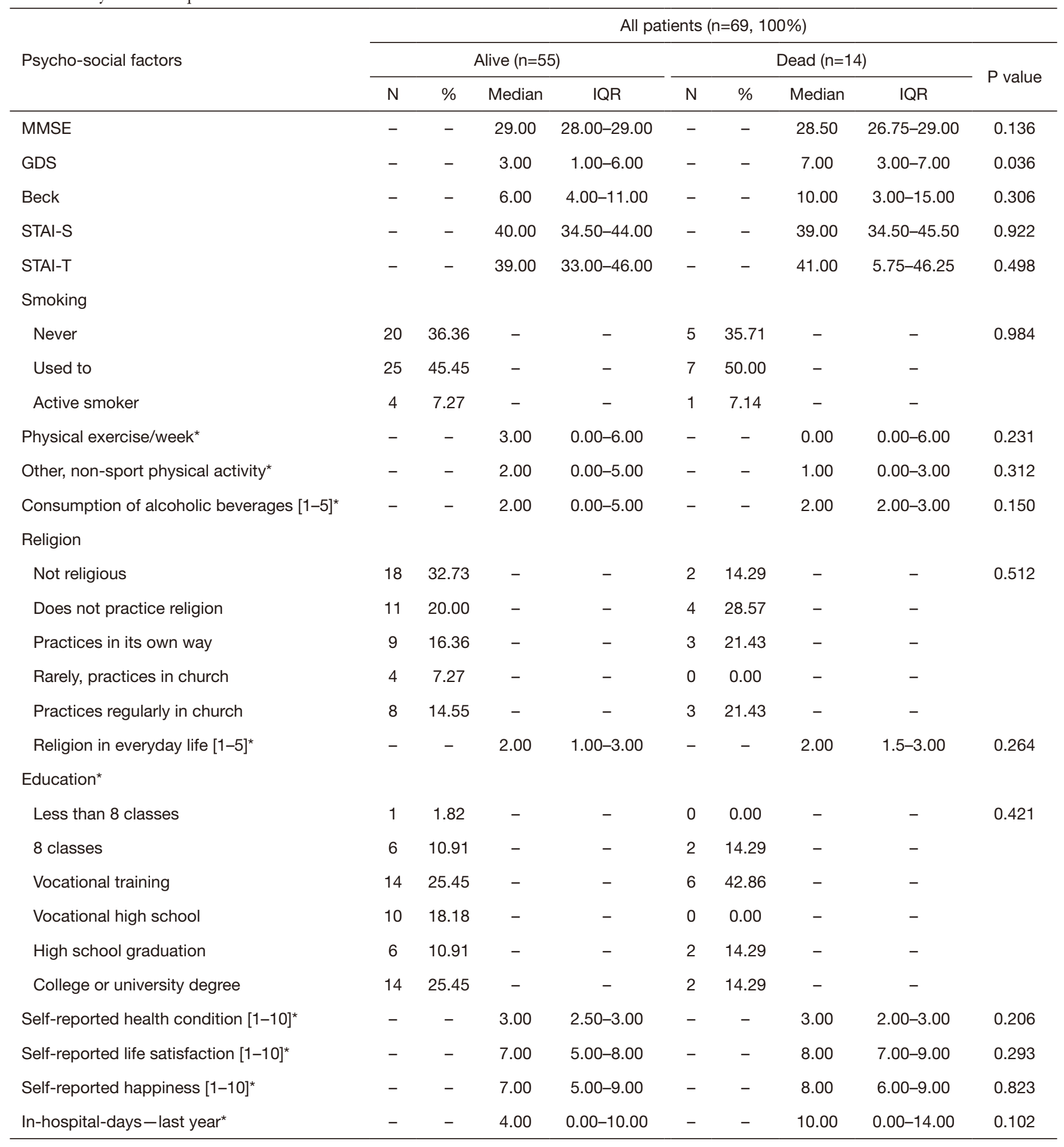

Chi square test was used for categorical variable statistics. *, continuous variable Mann-Whitney U test was used. Beck, Beck's Depression Inventory; IQR, interquartile range; MMSE, Mini-Mental State Examination; GDS, Geriatric Depression Scale; STAI-S, State-Trait Anxiety Inventory-State; STAI-T, State-Trait Anxiety Inventory-Trait. 
Table 5 Propensity score matched pairs balance calculations (cardiac surgery group vs. Hungarostudy group)

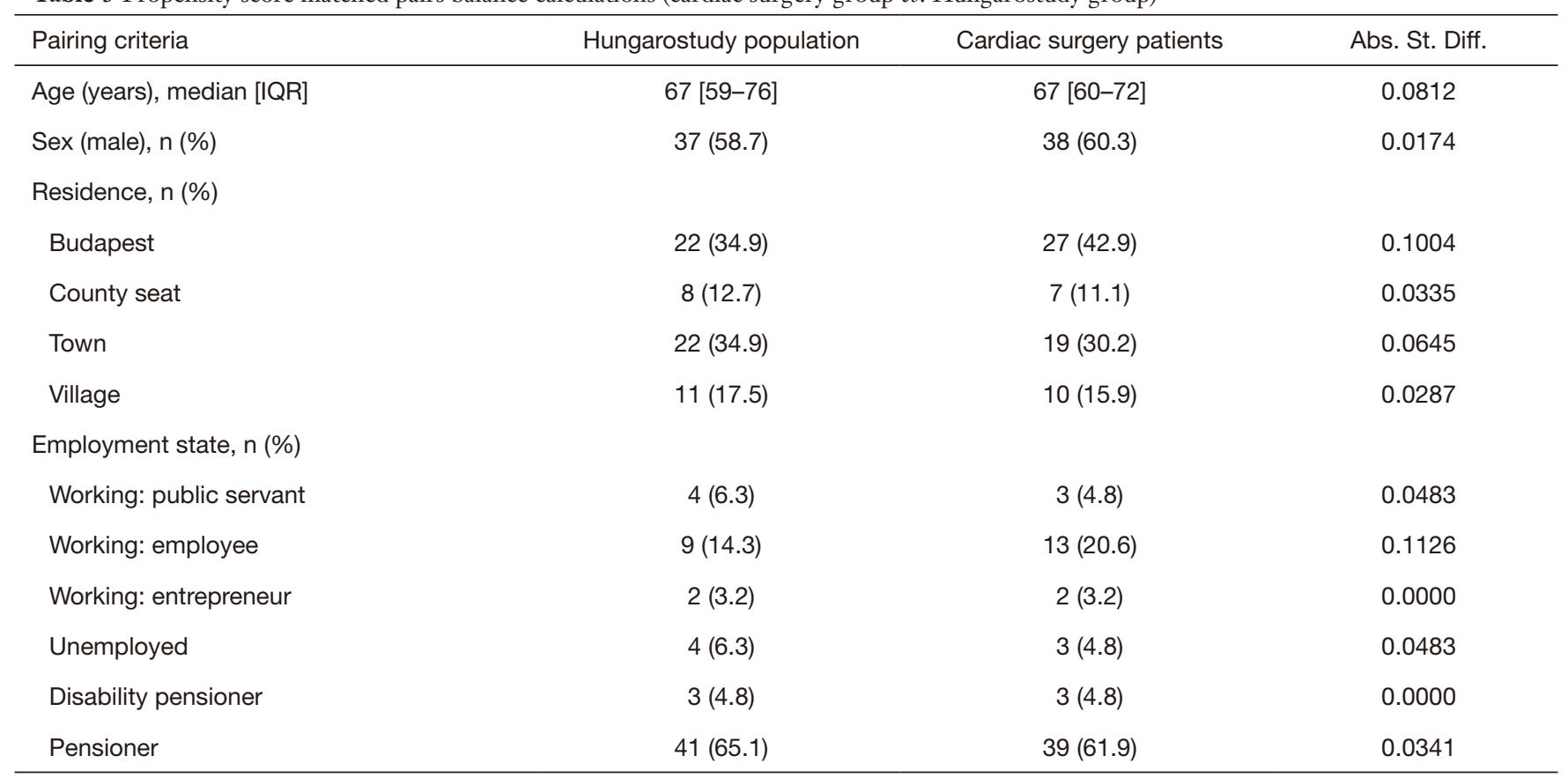

The propensity score matching is based on the variables shown above. IQR, interquartile range; Abs. St. Diff., absolute standardised difference.

(Nagi score), walking up or down a flight of stairs, or doing heavy work around the house (Rosow-Breslau score) were not significant. Therefore, we looked deeper into the other domains.

In accordance with our results, the CGA-based noncardiovascular domain was associated with increased mortality after cardiac surgery (48-50). The domain is a useful tool for summarizing the prediction of diseases, such as anxiety, arthritis, sensory impairment, etc., which are not assessed by the EuroSCORE II or the MELD score but characterize the general condition of frail patients. In contrast to our previous results in the vascular surgical population, cognitive dysfunction measured by MMSE was not associated with mortality among patients undergoing cardiac surgery. While the explanation for this requires more detailed investigation, we can initially conclude that despite the common precipitating factors, such as atherosclerosis, sedentary lifestyle, diabetes, and hypertension, the two populations differ from each other in cognitive dysfunction, incidence of stroke or TIA.

Nutrition, obesity and muscle weakness have also been used to assess frailty in older patients because these can cause infection, loss of physical function, and an elevated mortality rate (48). A reduction in physical function and activity, appetite and energy consumption may lead to the progression of malnutrition $(17,40,51)$. Calculation of BMI has been widely used in risk stratification with conflicting results. We have also found that BMI was not associated with mortality $(17,52)$. The serum albumin is a good indicator of malnutrition. Former studies have shown that low serum albumin is correlated with adverse outcomes after cardiovascular surgery (53). In patients who underwent cardiac surgery, a lower serum albumin level $(<3.5 \mathrm{~g} / \mathrm{dL})$ was associated with a higher risk of mortality $(\mathrm{P}<0.001)(54)$. Calculation of the nutritional state is an easy and inexpensive method, and can be used for optimal planning or rehabilitation in patients waiting for cardiac surgery.

Elderly people who have many comorbidities, such as cirrhosis, COPD and chronic renal failure, are prone to have a weaker nutritional status because of their physical impairments and drug-related factors. For example, the liver plays an important role in nutritional status through the production of albumin. Recently, hepatic dysfunction, regardless of the congestive or hypoxic nature, has been found to be an important predictor of mortality in advanced heart failure or after heart transplantation. Calculation of the MELD score has become an essential part of investigations before transplantation (55).

In patients with cirrhosis, the 30 -day mortality rate after cardiac surgery was higher (17). Our results are in 
Table 6 Propensity score matched pairs (cardiac surgery group vs. Hungarostudy group)

\begin{tabular}{|c|c|c|c|c|c|c|c|c|c|}
\hline \multirow{2}{*}{ Psycho-social factors } & \multicolumn{9}{|c|}{ All patients (63 pairs) } \\
\hline & $\mathrm{N}$ & $\%$ & Median & IQR & $\mathrm{N}$ & $\%$ & Median & IQR & $P$ value \\
\hline \multicolumn{10}{|l|}{ Smoke } \\
\hline Never & 29 & 46.03 & - & - & 35 & 55.56 & - & - & 0.014 \\
\hline Active smoker & 5 & 7.94 & - & - & 13 & 20.63 & - & - & \\
\hline Physical exercise/week* & - & - & 1.00 & $1.00-2.00$ & - & - & 1.00 & $1.00-1.00$ & 0.016 \\
\hline Other, non-sport physical activity* & - & - & 1.00 & $0.00-3.75$ & - & - & 3.00 & $1.00-4.00$ & 0.006 \\
\hline Consumption of alcoholic beverages $[1-5]^{*}$ & - & - & 2.00 & $1.00-3.00$ & - & - & 2.00 & $1.00-4.00$ & 0.836 \\
\hline Does not practice religion & 13 & 20.63 & - & - & 15 & 23.81 & - & - & \\
\hline Practices in its own way & 11 & 17.46 & - & - & 11 & 17.46 & - & - & \\
\hline Rarely, practices in church & 3 & 4.76 & - & - & 8 & 12.70 & - & - & \\
\hline Practices regularly in church & 11 & 17.46 & - & - & 8 & 12.70 & - & - & \\
\hline Religion in everyday life $[1-5]^{*}$ & - & - & 2.00 & $1.00-2.00$ & - & - & 1.00 & $1.00-3.00$ & 0.845 \\
\hline \multicolumn{10}{|l|}{ Education* } \\
\hline Less than 8 classes & 1 & 1.59 & - & - & 2 & 3.17 & - & - & 0.433 \\
\hline 8 classes & 8 & 12.70 & - & - & 14 & 22.22 & - & - & \\
\hline Live alone & 24 & 38.10 & - & - & 23 & 36.51 & - & - & 0.854 \\
\hline Financial difficulties & 3 & 4.76 & - & - & 11 & 17.46 & - & - & 0.023 \\
\hline Patient Health Quality* & - & - & 22.00 & $18.00-28.00$ & - & - & 21.00 & $16.50-25.00$ & 0.771 \\
\hline Self-reported health condition $[1-10]^{*}$ & - & - & 3.00 & $3.00-3.00$ & - & - & 3.00 & $3.00-4.00$ & 0.169 \\
\hline Self-reported life satisfaction $[1-10]^{*}$ & - & - & 7.00 & $5.00-8.00$ & - & - & 6.00 & $5.00-7.75$ & 0.195 \\
\hline Self-reported happiness $[1-10]^{*}$ & - & - & 7.00 & $5.00-8.00$ & - & - & 7.00 & $5.00-8.00$ & 0.510 \\
\hline In-hospital-days-last year* & - & - & 1.00 & $0.00-8.75$ & - & - & 0.00 & $0.00-0.00$ & $<0.001$ \\
\hline Caldwell Social Support Dimension Scale* & - & - & 21.00 & $19.00-25.00$ & - & - & 23.00 & $20.50-26.00$ & 0.052 \\
\hline Caldwell Social Support Dimension Scale-family* & - & - & 12.00 & $9.00-13.00$ & - & - & 12.00 & $10.00-14.00$ & 0.401 \\
\hline Caldwell Social Support Dimension Scale-other* & - & - & 9.00 & $6.00-11.00$ & - & - & 11.00 & $9.00-13.00$ & $<0.001$ \\
\hline
\end{tabular}

Chi square test was used for categorical variable statistics. *, continuous variable Mann-Whitney $U$ test was used. IQR, interquartile range. 
accordance with these findings. The albumin level and calculated GNRI score were associated with increased mortality. However, our results indicate that hepatic dysfunction, defined by MELD scores and the nutritional state, calculated by GNRI are independently associated with an increased risk for mid-term mortality. Additionally, a higher score in the nutritional part of the CGA also exhibited a strong relationship with mid- and long-term survival in cardiac surgical patients.

The GNRI is a numeric value derived from a formula based on serum albumin level, patient height and body weight. A value of less than 91 was defined as indicating the presence of malnutrition $(16,40)$. Low GNRI was found to be a risk factor for mid-term mortality after transcatheter aortic valve replacement (56) and a significant factor in the complicated postoperative period $(14,16)$. Low GNRI was reported to be associated with delayed or no rehabilitation after cardiac surgery (57).

Another aim of our study was to compare the cardiac surgery population with the general healthy Hungarian population. In our study, there were more ex-smokers than in the HS study (58). Multiple papers have highlighted the importance of social assistance in different clinical situations $(59,60)$.

In patients on the waiting list for surgery, low social support was associated with a lower likelihood of participation in rehabilitation than those with higher social support (59). The CSSDS measures the patient's social web structure. The bond of different interpersonal relationships and how much the participant can count on those around them were studied. Family, neighbours, colleagues and friends are represented in the questionnaire (61). Our findings suggest that the cardiac study population receives less social support outside of their families than the HS group, which would be an important factor in rehabilitation.

\section{Limitations of the study}

The main limitation of our current research is the relatively small number of patients. Completing the questionnaire took a relatively long time, which made it difficult to enroll patients in our research. We have also tried to distinguish between our previous studies about depression and anxiety, the HS epidemiological survey and the frailty assessment tools. First, we wanted to check the feasibility of comprehensive frailty tools in patients undergoing cardiac surgery. Second, we have tried to apply our recent previous experiences, and asked patients to complete both frailty and psychosocial questionnaires. Third, we compared our study population with the Hungarian survey. Although, in the case of psychological and sociological topics, tendencies play a major role.

\section{Conclusions}

We have found that the CGA-based non-cardiovascular score, Nutritional score and low GNRI were independently associated with long-term mortality after adjustment for EuroSCORE II. and postoperative complications. According to our results, older patients can safely undergo surgery, but proper risk stratification and or and/ or rehabilitation are needed to achieve optimal results. However, further large, multicenter and large database are needed to confirm our results.

\section{Acknowledgments}

The authors are grateful to the employees of the cardiac surgical ward of Heart and Vascular Centre, Semmelweis University, Budapest. We are grateful to Pal MaurovichHorvat, MD, $\mathrm{PhD}, \mathrm{MPH}$ for reviewing the statistical elements of our research. We are thankful for the American Journal Experts for the English language correction.

Funding: None.

\section{Footnote}

Reporting Checklist: The authors have completed the STROBE reporting checklist. Available at https://dx.doi. org/10.21037/apm-21-1015

Data Sharing Statement: Available at https://dx.doi. org/10.21037/apm-21-1015

Conflicts of Interest: All authors have completed the ICMJE uniform disclosure form (available at https://dx.doi. org/10.21037/apm-21-1015). The authors have no conflicts of interest to declare.

Ethical Statement: The authors are accountable for all aspects of the work in ensuring that questions related to the accuracy or integrity of any part of the work are appropriately investigated and resolved. The study was approved by the Scientific Research Ethics Committee, Semmelweis University, Budapest. The approval number 
is 250/2013 and ClinicalTrials.gov registration number is NCT02224222 (25/08/2014). Informed consent was obtained from all the study participants. All methods were conducted in accordance with the relevant guidelines and regulations (Declaration of Helsinki, the 2013 version).

Open Access Statement: This is an Open Access article distributed in accordance with the Creative Commons Attribution-NonCommercial-NoDerivs 4.0 International License (CC BY-NC-ND 4.0), which permits the noncommercial replication and distribution of the article with the strict proviso that no changes or edits are made and the original work is properly cited (including links to both the formal publication through the relevant DOI and the license). See: https://creativecommons.org/licenses/by-nc-nd/4.0/.

\section{References}

1. Nashef SA, Roques F, Sharples LD, et al. EuroSCORE II. Eur J Cardiothorac Surg 2012;41:734-44; discussion 744-5.

2. Berman M, Stamler A, Sahar G, et al. Validation of the 2000 Bernstein-Parsonnet score versus the EuroSCORE as a prognostic tool in cardiac surgery. Ann Thorac Surg 2006;81:537-40.

3. Czobor NR, Lehot JJ, Holndonner-Kirst E, et al. Frailty In Patients Undergoing Vascular Surgery: A Narrative Review Of Current Evidence. Ther Clin Risk Manag 2019;15:1217-32.

4. Darvall JN, Greentree K, Braat MS, et al. Contributors to frailty in critical illness: Multi-dimensional analysis of the Clinical Frailty Scale. J Crit Care 2019;52:193-9.

5. Székely A, Balog P, Benkö E, et al. Anxiety predicts mortality and morbidity after coronary artery and valve surgery--a 4-year follow-up study. Psychosom Med 2007;69:625-31.

6. Rockwood K, Song X, MacKnight C, et al. A global clinical measure of fitness and frailty in elderly people. CMAJ 2005;173:489-95.

7. Makary MA, Segev DL, Pronovost PJ, et al. Frailty as a predictor of surgical outcomes in older patients. J Am Coll Surg 2010;210:901-8.

8. Lee DH, Buth KJ, Martin BJ, et al. Frail patients are at increased risk for mortality and prolonged institutional care after cardiac surgery. Circulation 2010;121:973-8.

9. Ad N, Holmes SD, Halpin L, et al. The Effects of Frailty in Patients Undergoing Elective Cardiac Surgery. J Card Surg 2016;31:187-94.
10. Koh LY, Hwang NC. Frailty in Cardiac Surgery. J Cardiothorac Vasc Anesth 2019;33:521-31.

11. Afilalo J, Mottillo S, Eisenberg MJ, et al. Addition of frailty and disability to cardiac surgery risk scores identifies elderly patients at high risk of mortality or major morbidity. Circ Cardiovasc Qual Outcomes 2012;5:222-8.

12. Garrard JW, Cox NJ, Dodds RM, et al. Comprehensive geriatric assessment in primary care: a systematic review. Aging Clin Exp Res 2020;32:197-205.

13. Lee H, Lee E, Jang IY. Frailty and Comprehensive Geriatric Assessment. J Korean Med Sci 2020;35:e16.

14. Dent E, Hoogendijk EO, Visvanathan R, et al. Malnutrition Screening and Assessment in Hospitalised Older People: a Review. J Nutr Health Aging 2019;23:431-41.

15. Huang BT, Peng Y, Liu W, et al. Nutritional State Predicts All-Cause Death Independent of Comorbidities in Geriatric Patients with Coronary Artery Disease. J Nutr Health Aging 2016;20:199-204.

16. Bouillanne O, Morineau G, Dupont C, et al. Geriatric Nutritional Risk Index: a new index for evaluating at-risk elderly medical patients. Am J Clin Nutr 2005;82:777-83.

17. Unosawa $S$, Taoka $M$, Osaka $S$, et al. Is malnutrition associated with postoperative complications after cardiac surgery? J Card Surg 2019;34:908-12.

18. Duchnowski P, Hryniewiecki T, Kuśmierczyk M, et al. The usefulness of selected biomarkers in patients with valve disease. Biomark Med 2018;12:1341-6.

19. Golubovic M, Peric V, Stanojevic D, et al. Potential New Approaches in Predicting Adverse Cardiac Events One Month after Major Vascular Surgery. Med Princ Pract 2019;28:63-9.

20. Reddy HG, Shih T, Englesbe MJ, et al. Analyzing "failure to rescue": is this an opportunity for outcome improvement in cardiac surgery? Ann Thorac Surg 2013;95:1976-81; discussion 1981.

21. Roques F, Nashef SA, Michel P, et al. Risk factors and outcome in European cardiac surgery: analysis of the EuroSCORE multinational database of 19030 patients. Eur J Cardiothorac Surg 1999;15:816-22; discussion 822-3.

22. De Cassai A, Boscolo A, Tonetti T, et al. Assignment of ASA-physical status relates to anesthesiologists' experience: a survey-based national-study. Korean J Anesthesiol 2019;72:53-9.

23. Lima DFT, Cristelo D, Reis P, et al. Outcome prediction with Physiological and Operative Severity Score for the enumeration of Mortality and Morbidity score system in elderly patients submitted to elective surgery. Saudi J 
Anaesth 2019;13:46-51.

24. Szabó A, Tóth K, Nagy Á, et al. The effect of cognitive dysfunction on mid- and long-term mortality after vascular surgery. BMC Geriatr 2021;21:46.

25. Koivumaa-Honkanen H, Honkanen R, Viinamäki H, et al. Self-reported life satisfaction and 20-year mortality in healthy Finnish adults. Am J Epidemiol 2000;152:983-91.

26. Seese L, Sultan I, Gleason TG, et al. The Impact of Major Postoperative Complications on Long-Term Survival After Cardiac Surgery. Ann Thorac Surg 2020;110:128-35.

27. Guillén-Riquelme A, Buela-Casal G. Meta-analysis of group comparison and meta-analysis of reliability generalization of the State-Trait Anxiety Inventory Questionnaire (STAI). Rev Esp Salud Publica 2014;88:101-12.

28. Kopp MS. Psychophysiological characteristics of anxiety patients and controls. Psychother Psychosom 1989;52:74-9.

29. Robinson BE, Kelley L. Concurrent validity of the Beck Depression Inventory as a measure of depression. Psychol Rep 1996;79:929-30.

30. Pálinkás A, Sándor J, Papp M, et al. Associations between untreated depression and secondary health care utilization in patients with hypertension and/or diabetes. Soc Psychiatry Psychiatr Epidemiol 2019;54:255-76.

31. Devins GM. Using the illness intrusiveness ratings scale to understand health-related quality of life in chronic disease. J Psychosom Res 2010;68:591-602.

32. Yesavage JA, Brink TL, Rose TL, et al. Development and validation of a geriatric depression screening scale: a preliminary report. J Psychiatr Res 1982-1983;17:37-49.

33. Crum RM, Anthony JC, Bassett SS, et al. Populationbased norms for the Mini-Mental State Examination by age and educational level. JAMA 1993;269:2386-91.

34. Hinz A, Ernst J, Glaesmer H, et al. Frequency of somatic symptoms in the general population: Normative values for the Patient Health Questionnaire-15 (PHQ-15). J Psychosom Res 2017;96:27-31.

35. Soldatos CR, Dikeos DG, Paparrigopoulos TJ. Athens Insomnia Scale: validation of an instrument based on ICD10 criteria. J Psychosom Res 2000;48:555-60.

36. Leavitt MB. Family recovery after vascular surgery. Heart Lung 1990;19:486-90.

37. Kao TA, Caldwell CH. Family Efficacy within Ethnically Diverse Families: A Qualitative Study. Fam Process 2017;56:217-33.

38. Susanszky É, Székely A. Hungarostudy 2013. Database. Available online: http://www.hungarostudy.hu/, accessed
25 Oct 2020.

39. Susányszky É, Székely A. A Hungarostudy 2013 felmérés módszertana. In: Susánszky É. Szántó Zs. editors. Magyar lelkiállapot [The Hungarian mental state]. Budapest: Semmelweis, 2013:13-21.

40. Luo H, Yang H, Huang B, et al. Geriatric Nutritional Risk Index (GNRI) Independently Predicts Amputation Inchronic Criticallimb Ischemia (CLI). PLoS One 2016;11:e0152111.

41. Yenibertiz D, Cirik MO. The comparison of GNRI and other nutritional indexes on short-term survival in geriatric patients treated for respiratory failure. Aging Clin Exp Res 2021;33:611-7.

42. Fried LP, Tangen CM, Walston J, et al. Frailty in older adults: evidence for a phenotype. J Gerontol A Biol Sci Med Sci 2001;56:M146-56.

43. Scarborough JE, Bennett KM, Englum BR, et al. The impact of functional dependency on outcomes after complex general and vascular surgery. Ann Surg 2015;261:432-7.

44. Forman DE, Rich MW, Alexander KP, et al. Cardiac care for older adults. Time for a new paradigm. J Am Coll Cardiol 2011;57:1801-10.

45. Keeney T, Jette DU, Cabral H, et al. Frailty and Function in Heart Failure: Predictors of 30-Day Hospital Readmission? J Geriatr Phys Ther 2021;44:101-7.

46. Xu W, Cai Y, Liu H, et al. Frailty as a predictor of allcause mortality and readmission in older patients with acute coronary syndrome : A systematic review and metaanalysis. Wien Klin Wochenschr 2020;132:301-9.

47. Wahl TS, Graham LA, Hawn MT, et al. Association of the Modified Frailty Index With 30-Day Surgical Readmission. JAMA Surg 2017;152:749-57.

48. Takagi H, Ando T, Umemoto T, et al. Perioperative depression or anxiety and postoperative mortality in cardiac surgery: a systematic review and meta-analysis. Heart Vessels 2017;32:1458-68.

49. Parhar K, Zochios V. Outcomes of Patients With COPD Undergoing Cardiac Surgery: Don't Hold Your Breath. J Cardiothorac Vasc Anesth 2018;32:2246-7.

50. Lysak N, Bihorac A, Hobson C. Mortality and cost of acute and chronic kidney disease after cardiac surgery. Curr Opin Anaesthesiol 2017;30:113-7.

51. Dhesi JK, Lees NP, Partridge JS. Frailty in the perioperative setting. Clin Med (Lond) 2019;19:485-9.

52. Tokarek TA, Dziewierz A, Sorysz D, et al. The obesity paradox in patients undergoing transcatheter aortic valve implantation: is there any effect of body mass index on 
survival? Kardiol Pol 2019;77:190-7.

53. Nochioka K, Sakata Y, Takahashi J, et al. Prognostic impact of nutritional status in asymptomatic patients with cardiac diseases: a report from the CHART-2 Study. Circ J 2013;77:2318-26.

54. Montazerghaem H, Safaie N, Samiei Nezhad V. Body Mass Index or Serum Albumin Levels: Which is further Prognostic following Cardiac Surgery? J Cardiovasc Thorac Res 2014;6:123-6.

55. Harjola VP, Mullens W, Banaszewski M, et al. Organ dysfunction, injury and failure in acute heart failure: from pathophysiology to diagnosis and management. A review on behalf of the Acute Heart Failure Committee of the Heart Failure Association (HFA) of the European Society of Cardiology (ESC). Eur J Heart Fail 2017;19:821-36.

56. Shibata K, Yamamoto M, Kano S, et al. Importance of Geriatric Nutritional Risk Index assessment in patients undergoing transcatheter aortic valve replacement. Am Heart J 2018;202:68-75.

57. Ogawa M, Izawa KP, Satomi-Kobayashi S, et al. Poor

Cite this article as: Tóth K, Szabó A, Nagy Á, Szabó D, Szécsi B, Eke C, Sándor Á, Susánszky É, Holndonner-Kirst E, Merkely B, Gál J, Székely A. Preoperative nutritional state is associated with mid- and long-term mortality after cardiac surgery. Ann Palliat Med 2021;10(11):11333-11347. doi: 10.21037/ apm-21-1015 preoperative nutritional status is an important predictor of the retardation of rehabilitation after cardiac surgery in elderly cardiac patients. Aging Clin Exp Res 2017;29:283-90.

58. Konkolÿ Thege B, Bachner YG, Kushnir T, et al. Relationship between meaning in life and smoking status: results of a national representative survey. Addict Behav 2009;34:117-20.

59. Pushkarev G, Kuznetsov V, Yaroslavskaya E, et al. Social support for patients with coronary artery disease after percutaneous coronary intervention. J Psychosom Res 2019;119:74-8.

60. Aliche JC, Ifeagwazi CM, Chukwuorji JC, et al. Roles of Religious Commitment, Emotion Regulation and Social Support in Preoperative Anxiety. J Relig Health 2020;59:905-19.

61. Devins GM, Binik YM, Hutchinson TA, et al. The emotional impact of end-stage renal disease: importance of patients' perception of intrusiveness and control. Int J Psychiatry Med 1983-1984;13:327-43. 